\title{
Erratum: Di-boson signatures as standard candles for partial compositeness
}

\author{
Alexander Belyaev, ${ }^{a, b}$ Giacomo Cacciapaglia, ${ }^{c}$ Haiying Cai, ${ }^{c}$ Gabriele Ferretti, ${ }^{d}$ \\ Thomas Flacke, , $f$ Alberto Parolini ${ }^{f}$ and Hugo Serodio ${ }^{f, g}$ \\ ${ }^{a}$ School of Physics \& Astronomy, University of Southampton, \\ Southampton, U.K. \\ ${ }^{b}$ Particle Physics Department, Rutherford Appleton Laboratory, \\ Chilton, Didcot, Oxon OX11 0QX, U.K. \\ ${ }^{c}$ Univerity of Lyon, Université Lyon 1, \\ CNRS/IN2P3, IPNL, F-69622, Villeurbanne, France \\ ${ }^{d}$ Department of Physics, Chalmers University of Technology, \\ Fysikgården, 41296 Göteborg, Sweden \\ ${ }^{e}$ Center for Theoretical Physics of the Universe, Institute for Basic Science (IBS), \\ Daejeon, 34051, Korea \\ ${ }^{f}$ Department of Physics, Korea University, \\ Seoul 136-713, Korea \\ ${ }^{g}$ Department of Astronomy and Theoretical Physics, \\ Lund University, SE-223 62 Lund, Sweden \\ E-mail: a.belyaev@soton.ac.uk, g.cacciapaglia@ipnl.in2p3.fr, \\ hcai@ipnl.in2p3.fr, ferretti@chalmers.se, flacke@ibs.re.kr, \\ parolini85@kias.re.kr, hugo.serodio@thep.lu.se
}

ERRATUM TO: JHEP01(2017)094

ArXiv EPRINT: 1610.06591

The sign of $C_{t}$ in appendix C, eqs. (C.1), (C.7), (C.8) needs to be reversed. The correct relations for eqs. (C.1), (C.7), (C.8) are:

$$
\mathcal{M}_{\text {top }}^{\text {odd }}=-\frac{\epsilon_{\mu \nu \rho \sigma} \epsilon^{\mu}(\vec{k}) \epsilon^{\nu}(\vec{p}) k^{\rho} p^{\sigma}}{4 \pi^{2}} \frac{C_{t}}{f_{\pi}}\left[c_{1} \widetilde{C}_{0}\left(R_{p}, R_{k}, R_{\pi} ; \xi\right)+c_{2} \widetilde{C}_{1}\left(R_{p}, R_{p}, R_{\pi} ; \xi\right)\right],
$$




$$
\begin{aligned}
\frac{\Gamma_{\mathrm{WZW}+\text { top }}\left(\pi_{0} \rightarrow g g\right)}{\Gamma_{\mathrm{WZW}}\left(\pi_{0} \rightarrow g g\right)}=\left|1-\frac{C_{t}}{\kappa_{g}} \widetilde{C}_{0}\left(0,0, R_{\pi_{0}} ; 1\right)\right|^{2}, \\
\frac{\Gamma_{\mathrm{WZW}+\text { top }}\left(\pi_{0} \rightarrow \gamma \gamma\right)}{\Gamma_{\mathrm{WZW}}\left(\pi_{0} \rightarrow \gamma \gamma\right)}=\left|1-\frac{8}{3} \frac{C_{t}}{\kappa_{B}+\kappa_{W}} \widetilde{C}_{0}\left(0,0, R_{\pi_{0}} ; 1\right)\right|^{2}, \\
\frac{\Gamma_{\mathrm{WZW}+\text { top }}\left(\pi_{0} \rightarrow W^{+} W^{-}\right)}{\Gamma_{\mathrm{WZW}}\left(\pi_{0} \rightarrow W^{+} W^{-}\right)}=\left|1-\frac{3}{2} \frac{C_{t}}{\kappa_{W}} \widetilde{C}_{1}\left(R_{W}, R_{W}, R_{\pi_{0}} ; \xi\right)\right|^{2}, \\
\frac{\Gamma_{\mathrm{WZW}+\text { top }}\left(\pi_{0} \rightarrow Z \gamma\right)}{\Gamma_{\mathrm{WZW}}\left(\pi_{0} \rightarrow Z \gamma\right)}=\left|1-\frac{2}{c_{W}^{2}}\left(\frac{1}{2}-\frac{4 s_{W}^{2}}{3}\right) \frac{C_{t}}{\kappa_{W}-t_{W}^{2} \kappa_{B}} \widetilde{C}_{0}\left(R_{Z}, 0, R_{\pi_{0}} ; 1\right)\right|^{2}, \\
\frac{\Gamma_{\mathrm{WZW}+\text { top }}\left(\pi_{0} \rightarrow Z Z\right)}{\Gamma_{\mathrm{WZW}}\left(\pi_{0} \rightarrow Z Z\right)}=\mid 1-\frac{C_{t}}{\kappa_{W}+t_{W}^{4} \kappa_{B}}\left(\frac{3}{2 c_{W}^{4}} \widetilde{C}_{1}\left(R_{Z}, R_{Z}, R_{\pi_{0}} ; 1\right)\right. \\
\left.\left.\frac{\Gamma_{\mathrm{WZW}+\text { top }}\left(\pi_{8} \rightarrow g g\right)}{\Gamma_{\mathrm{WZW}}\left(\pi_{8} \rightarrow g g\right)}=\mid 1-\frac{4 s_{W}^{2}}{3}-1\right) \widetilde{C}_{0}\left(R_{Z}, R_{Z}, R_{\pi_{0}} ; 1\right)\right)\left.\right|^{2}, \\
\frac{\Gamma_{\mathrm{WZW}+\text { top }}\left(\pi_{8} \rightarrow g \gamma\right)}{\Gamma_{\mathrm{WZW}}\left(\pi_{8} \rightarrow g \gamma\right)}=\left|1-\frac{4}{3} \frac{C_{t}}{\kappa_{g B}} \widetilde{C}_{0}\left(0,0, R_{\pi_{8}} ; 1\right)\right|^{2}, \\
\frac{\Gamma_{\mathrm{WZW}+\text { top }}\left(\pi_{8} \rightarrow g Z\right)}{\Gamma_{\mathrm{WZW}}\left(\pi_{8} \rightarrow g Z\right)}=\left|1-\frac{1}{2 s_{W}^{2}}\left(\frac{1}{2}-\frac{4 s_{W}^{2}}{3}\right) \frac{C_{t}}{\kappa_{g B}} \widetilde{C}_{0}\left(R_{Z}, 0, R_{\pi_{8}} ; 1\right)\right|^{2},
\end{aligned}
$$

The analysis in the main text is unchanged since we neglected loop corrections for large pNGB masses, there.

Open Access. This article is distributed under the terms of the Creative Commons Attribution License (CC-BY 4.0), which permits any use, distribution and reproduction in any medium, provided the original author(s) and source are credited. 